\title{
Semantic effects in encoding specificity: A levels of processing approach
}

\author{
ERIC GOLDSTEIN, JOHN C. SCHMITT, and C. JAMES SCHEIRER \\ State University of New York at Binghamton, Binghamton, New York 13901
}

\begin{abstract}
The semantic characteristics of study and retrieval contexts were varied in a cued recall paradigm. In the first experiment, ambiguous words (homographs) were modified at input and output by identical adjectives or by different adjectives reflecting either the same meaning or a different meaning. The results supported the principle of encoding specificity (Thomson \& Tulving, 1970), but only for identical input and output cues; the results showed no facilitation of similar semantic context on recall. The second experiment used a depth-of-processing manipulation to show that the results predicted by a semantic interpretation of encoding specificity are obtainable, but only when subjects are induced to process deeply. The implications of these data for the generality of encoding specificity are discussed.
\end{abstract}

The study of retrieval processes in cued recall has been the source of much literature in the last decade. A number of explanations have been formulated in an attempt to explain the effectiveness of cues, the most explicit being the encoding specificity principle (Thomson \& Tulving, 1970; Tulving \& Thomson, 1973) and the generation-recognition hypothesis (Anderson \& Bower, 1972; Bahrick, 1969, 1970). The encoding specificity principle as defined by Thomson and Tulving (1970) states that "no cue, however strongly associated with the to-be-remembered item or otherwise related to it, can be effective unless the to-be-remem. bered item is specifically encoded with respect to that cue at the time of its storage" (p. 255). Encoding specificity is concerned with the processes involved in the storage of items, that is, in how the subject encodes the information presented. Facilitation of retrieval by extralist cues will depend on the nature of these encoding processes. Results supporting encoding specificity are abundant. Thomson and Tulving (1970) found that if subjects studied items in the presence of weak associates at the input stage (e.g.. ground COLD) and were then given a strong associative cue at output (e.g., hot-COLD), recall was only slightly facilitated relative to free recall. If the subject was given the same weak associate at the output stage, however, recall improved markedly.

Thomson and Tulving explain these results in the framework of encoding specificity: Although the cue ground is only weakly associated with the to-be-remem-

Portions of this paper were presented at the annual meeting of the Psychonomic Society, St. Louis, November 1976, and at the Eastern Psychological Association, Boston, April 1977. Experiment 1 was based on the senior honors of the first author. Requests for reprints should be addressed to C. James Scheirer, Department of Psychology, State University of New York at Binghamton, Binghamton, New York 13901. John Schmitt is now at Bell Telephone Laboratories, Holmdel, New Jersey. bered (TBR) item COLD in a normative sense, it is part of the encoded memory trace. It is this specific encoding which facilitates recall at output. In further studies Tulving and Thomson (1973) have replicated and extended the results of their previous research. In general, an encoding specificity position differs from a generation-recognition position in its predictions of what information will be effective in retrieval. Generation recognition relies predominantly on normative associative information, while encoding specificity assumes only "encoding-specific" information will be effective, independent of its normative properties. More recent formulations of generation recognition have built in the encoding specificity hypothesis, making the predictions of the two positions similar (cf. Reder, Anderson, \& Bjork, 1974, p. 654).

One important statement that Tulving and Thomson (1973) have made is that the processing subsumed under the encoding specificity principle occurs in episodic and not semantic memory. The difference between these is that "episodic memory is concerned with storage and retrieval of temporally dated, spatially located, and personally experienced events or episodes, and temporal-spatial relations among such events....Semantic memory is the system concerned with storage and utilization of knowledge about words and concepts, their properties, and interrela. tions" (p. 354). One major problem with the episodicsemantic distinction, however, is the question of how these memories interact to produce the episodic trace. We may distinguish between two classes of predictions, relating to "nominal" or "semantic" interpretations of encoding specificity.

A nominal interpretation of encoding specificity would suggest that information is stored in memory in a relatively "isolated" form. By isolated, we mean that the stored trace contains little information other than the word's external context and is not greatly 
elaborated by its "meaning." Following the analytic approach to cuing suggested by Tulving and Bower (1974) and Tulving and Watkins (1975), for example, if the memory were retrievable only through presentation of aspects of the experimental context, we would infer an encoding of nominal features only. Such an interpretation was suggested by Tulving (1974) to explain certain aspects of the phenomenon of recognition failure of recallable words (Tulving \& Thomson, 1973), an interpretation discussed by Martin (1975).

A semantic interpretation of encoding specificity, on the other hand, suggests "richer" encoding of items in which substantial amounts of information are drawn from semantic memory and become part of the memory trace (Craik \& Tulving, 1975). This semantic enrichment corresponds to processes suggested by numerous theorists, including Craik and Lockhart (1972, "Type 2 processing"), Craik and Watkins (1973, "active rehearsal"), and Woodward, Bjork, and Jongeward (1973, "elaborative encoding"). By drawing a nominal/semantic distinction, we do not wish to imply, of course, that there is no involvement of semantic memory in nominal encoding. A subject must, for example, recognize that the items presented are words, that the items are presented in a language he speaks, and that the items correspond to some concept in that language. The interpretations differ chiefly in the greater amount of semantic information implied by a semantic interpretation.

Some support for a semantic interpretation has also been found. Reder et al. (1974) found that highfrequency words, which presumably have many meanings or "senses," are more sensitive to the recognition failure effect. This implies that particular meanings of even nonhomographic words are encoded, which demands semantic memory assumptions. This argument has been made even more strongly by Martin (1975), who suggests that different senses of a word should be considered different entities for the purpose of encoding specificity. More direct evidence is that of Barclay, Bransford, Franks, McCarrell, and Nitsch (1974). These researchers found that, depending on the attribute biased at input, cues emphasizing the weight of a PIANO ("something heavy") and the sound of a PIANO ("something with a nice sound") were differentially effective in recall. Apparently, sentences accompanying the items at input were sufficient to produce a highly specific involvement of semantic memory in the encoding process, so that only semantic information could be effective in retrieval.

The evidence most relevant to a semantic interpretation of the Thomson and Tulving (1970) paradigm, however, arises from studies by Hunt and Ellis (1974) and Light and Carter-Sobell (1970). In the Light and Carter-Sobell study, homographic nouns were accom- panied at input by cues biasing only one of the noun's meanings (e.g., strawberry-JAM). Recognition was tested either in the presence of this cue or in the presence of other cues biasing either the same meaning (raspberry) or a different meaning (traffic). Cuing with the same adjective produced recognition which was superior to that obtained with changed cues. However, maintaining the meaning of a TBR word with a nominally different modifier facilitated recognition relative to changing the meaning. The encoding specificity principle, then, must be extended to include semantic features of TBR words as well as their nominal features, at least within the bounds of this experiment. This apparently is the conclusion reached by Craik and Tulving (1975) when they state that "at encoding the stimulus is interpreted in terms of the system's structured record of past learning, that is, knowledge of the world or 'semantic memory'" (p. 291).

However, Hunt and Ellis (1974) failed to find a difference between same-meaning and different-meaning modifiers in a recognition paradigm. In this study the meaning of the homographic nouns was biased only with adjective modifiers; in the Light and CarterSobell (1970) study the meanings of nouns were biased by embedding them in sentences. As Hunt and Ellis point out, this difference in procedure may be critical to the different results obtained in the two studies because of the "more complete biasing of one meaning of the homograph" (Hunt \& Ellis, 1974, p. 157) in the Light and Carter-Sobell study.

It is clear that the issue of semantic involvement in encoding specificity is not resolved, either theoretically or empirically. Since the modal procedure in this area is the cued recall paradigm, it is particularly important to resolve this ambiguity here. The present experiments are attempts to investigate the involvement of semantic components in cued recall, using design procedures similar to those of Hunt and Ellis (1974) and Light and Carter-Sobell (1970). Homographs are employed as TBR words in order to change the meaning of words while maintaining the nominal TBR word itself. These homographs are paired with adjective modifiers which determine the semantic context at both input and output. The orthogonal manipulation of all possible input-output conditions, therefore, allows for specific predictions for nominal vs. semantic interpretations of the encoding specificity principle.

\section{EXPERIMENT 1}

\section{Method}

Materials. Forty-four homographs selected from word lists compiled by Cramer (1970) and by Whitford (1966) were used as TBR stimuli. The cues used were adjectives which biased the meaning of each homograph, as determined by agreement among the experimenters.

Design. Two lists of 22 TBR homographs were presented to 
Table 1

Input and Output Conditions (Contexts) Illustrating the TBR Homograph, JAM

\begin{tabular}{llcccc}
\hline & \multicolumn{5}{c}{ Output } \\
\cline { 2 - 6 } \multicolumn{1}{c}{ Input } & $\begin{array}{l}\text { straw- } \\
\text { berry }\end{array}$ & $\begin{array}{c}\text { rasp- } \\
\text { berry }\end{array}$ & traffic & log & no cue \\
\hline strawberry & SW & SM & DM & DM & C-0 \\
raspberry & SM & SW & DM & DM & C-0 \\
traffic & DM & DM & SW & SM & C-0 \\
log & DM & DM & SM & SW & C-0 \\
no cue & $0-C$ & 0-C & $0-C$ & $0-C$ & $0-0$ \\
\hline
\end{tabular}

Note-Entries in cells represent the functional conditions of the design used in data analysis (see text).

subjects for study, with a 5-min interval between testing of List 1 and presentation of List 2 . A completely randomized 5 by 5 between-groups design was employed to vary the input and output contexts. The design is illustrated in Table 1 and may be clarified through the use of an example TBR word, JAM. At input, JAM was presented with the study cue strawberry or raspberry (biasing one meaning), or with traffic or $\log$ (biasing another), or with no study cue. Recall was in the presence of one of the five cue conditions. The 25 groups, then, represent the factorial combination of the 5 (input) by 5 (output) conditions for that TBR word.

For the purposes of later statistical analysis the groups derived from the 5 by 5 design were collapsed into six functional conditions. Condition SW (same word) consisted of items in which an identical adjective modifier appeared at input and output. Condition SM (same meaning) consisted of items in which the adjective modifier was nominally different at output but biased the same meaning as the adjective presented at input. Condition DM (different meaning) consisted of items in which the adjective was presented at input. Condition $\mathrm{C}-0$ (cue at input, no cue at output) consisted of items presented for study in the presence of an adjective modifier but recalled in the absence of any modifier. Condition $0-C$ (no cue at input, cue at output) consisted of items presented with no adjective at input but with adjectives at output; Condition 0-0 consisted of items both presented and recalled in the absence of an adiective modifier. Blocking of the conditions in this manner produced unequal group sizes (see Table 2), making the analysis somewhat unwieldly, but it did assure equal representation of all inputoutput conditions.

Procedure. For both List 1 and List 2, all subjects were shown 22 homographs, projected on a screen in the front of the room at a rate of $5 \mathrm{sec}$ per item. The subjects were instructed that they would see a list of capitalized words and would have to remember as many of the words as possible. They were told that each word could be accompanied by another word in lowercase letters, located above the TBR word, which might be used as an aid in remembering the capitalized word. It was stressed that the capitalized word (homograph) was to be recalled, not the lowercase words.

Immediately after the study trial the subjects received a recall sheet and were told to write down as many of the capitalized words as they could. The recall period was $2 \mathrm{~min}$ Since Santa and Lamwers (1974) have argued that subjects may become confused when new cue words appear at recall (but see Wiseman \& Tulving, 1975), subjects were instructed that the recall sheet might contain some of the lowercase words seen in the study trial. They were also told that there might be new cue words not previously seen, and that these words might have some associative relationship to the capitalized words. Cue words were listed on the recall sheet, but subjects were instructed that they could record responses any place on the protocol they pleased. In later scoring of the protocols, items were tabulated as correct if they appeared anywhere on the page, irrespective of whether they were paired with the "appropriate" cue. The lenient scoring criterion was used to allow comparisons between output conditions which were or were not cued. To require that responses be written next to the associated cue would mean placing a constraint on the recall of cued subjects but not on uncued subjects.

Subjects. The subjects, 150 introductory psychology students at the State University of New York at Binghamton, served in order to fulfill a course requirement. Assignment to the various conditions was randomly determined by order of arrival at the laboratory. The subjects were tested in groups, the size of which varied from 1 to 21 , with each group receiving the same input condition. Subjects within each input group were subdivided into five groups of approximately the same size, and each of the groups received recall sheets corresponding to one of the five output conditions illustrated in Table 1. Upon completion of the design, there were six subjects in each of the 25 input by output conditions.

\section{Results}

A 6 (conditions) by 2 (lists) analysis of variance was first performed to test for List $1 /$ List 2 differences. List position had no overall effect $(F<1)$, nor did it interact with conditions $[F(5,144)=1.34, \mathrm{p}>.25]$. For this reason all subsequent analyses were performed on data pooled across both lists. For all subsequent analyses a level of significance of $p<.05$ was chosen; the mean square error for all $F$ values was 11.73 .

Mean recall scores for the six groups are presented in Table 2. Performance was significantly greater when identical items were presented at input and output (Condition SW) than for all other conditions combined $[F(1,144)=74.36]$. In particular, recall in Condition SW exceeded recall in Condition SM $[F(1,144)=52.14]$. This finding appears to be at variance with the predictions of an unrefined generationrecognition hypothesis since the cues presented at recall in the two conditions were identical; the conditions differed only with respect to the cues present at input. In spite of this identity between input cues, the magnitude of the differences in recall between Conditions SW and SM suggests that nominal similarity is much more important than semantic similarity in facilitating recall.

A more direct test of the relative importance of semantic and nominal factors in cuing is in the comparison between Condition SM (different output item/ same meaning) and Condition DM (different output item/different meaning). Recall in these conditions did not differ $(F<1)$. This suggests that all of the

Table 2

Means and Standard Deviations of Recall Scores (Number of Items Recalled) and ns for Each Condition in Experiment 1

\begin{tabular}{lcccccc}
\hline & \multicolumn{6}{c}{ Condition } \\
\cline { 2 - 7 } & SW & SM & DM & C-0 & 0-C & $0-0$ \\
\hline Mean & 17.38 & 8.88 & 7.95 & 8.96 & 9.69 & 12.08 \\
SD & 2.48 & 2.16 & 2.47 & 2.04 & 2.45 & 2.74 \\
n & 24 & 24 & 48 & 24 & 24 & 6 \\
\hline
\end{tabular}


effect of changing cues is associated with the nominal features of the stimulus item (SW vs. SM and DM) and that little or none is associated with the semantic features (SM vs. DM).

It is of considerable interest to note that recall in Condition 0-0 was greater than recall in all of the changed input-output contexts (SM, DM, C-0, 0-C) conditions combined $[F(1,144)=12.00]$. This result, taken in conjunction with the fact that conditions in which a cue was changed did not differ among themselves $\left(\operatorname{LSD}_{\alpha=.05}=2.32\right)$, suggests that any change in cue produces a decrement in performance relative to conditions in which subjects are left to their own devices. A very similar effect has been recently discussed by Ehrlich and Philippe (1976). They propose a "negative encoding" phenomenon, where the effect of an input cue is to modulate, in either a positive or negative direction, the effects of a cue at output and its associative strength with the target item. A similar finding was reported by Hunt and Ellis (1974) for recognition. Thomson and Tulving (1970), however, have shown that strong associative cues can facilitate recall relative to a free recall situation.

\section{Discussion}

The results of Experiment 1 clearly support the principle of encoding specificity. When subjects see the identical cue at input and output, recall is facilitated relative to all other conditions. The effectiveness of cuing per se is apparent in the comparison between Condition SW and Condition 0-0. Furthermore, since similar cues were less effective than identical cues in facilitating recall, it appears that any change in the context of the TBR words at time of recall is detrimental. The more striking results, however, are the lack of differences among groups in which any change between input and output cues occurred (Conditions SM, DM, C-0, 0-C) and their inferiority to free recall (Condition 0-0).

It might have been expected from prior results that performance in at least some of the groups would exceed performance in the free recall group. For example, while Thomson and Tulving (1970) found that recall was poorer when a weak cue was presented at output than when no cue was presented, they found that recall was better in the presence of a high normative associate. It must be remembered, however, that the cues in this study were chosen for their ability to bias meaning, not as high normative associates, and therefore all of the cues might have acted as weak associates in the Thomson and Tulving sense.

The comparison directly related to the role of semantic factors in cuing is that between Conditions SM and DM. The lack of difference may indicate that encoding is restricted to the nominal features of the cues, or at least that nominal features are much more important than semantic features in cued recall. Semantic features, to the extent that they overlap with the SM cues used in this experiment, afforded no advantage in recall relative to other, unrelated cues.

\section{EXPERIMENT 2}

A difficult problem potentially limiting interpretation of Experiment 1 relates to its conceptual design. The finding in favor of a nominal interpretation of encoding specificity is essentially one of no differences (between Conditions SM and DM). This null-hypothesis aspect of the design places the burden of proof on the alternative, semantic, interpretation. If we are to argue that little or no semantic memory involvement is present under normal cued recall conditions, it should be possible to show semantic effects under conditions where subjects are induced to process semantically. Experiment 2 did this by using a depth-of-processing (cf. Craik \& Lockhart, 1972) instructional manipulation.

\section{Method}

Materials. There were several potential problems with Experiment 1. First, the list materials (homographs and modifiers) were developed through consensus among the experimenters, and included some cues which were themselves homographic. These were eliminated in preparation for Experiment 2. Second, we had an independent group of subjects generate the lists used in Experiment 2. These subjects were given a list of homographs, told that each had two meanings, and asked to generate up to four modifiers biasing each meaning. Only nouns which elicited exactly two meanings were subsequently used, and cues were selected on the basis of their frequency of generation by this group of subjects (minimum frequency: 15\% of sample). Third, a second group of subjects was used to assure that each cue was effectively related to only one TBR noun. This was done by administering a matching test, in which subjects matched adjectives to the noun that "made the most sense" or "fit the best." All cuetarget pairs subsequently used were correctly matched by $90 \%$ or more of this sample. The resulting list of 27 homographs and sets of cues is presented in Table $3 .^{1}$

Design. The subjects, 192 introductory psychology students, were randomly assigned to 24 conditions, representing the factorial combination of the six cuing conditions described in Experiment 1 and the four instructional conditions. Subjects were tested in groups, with all members of a group receiving the same input list and orienting instruction. Across the experiment, and in contrast to Experiment 1, input and output lists were scheduled such that equal numbers of subjects were in the six functional cuing conditions. All other aspects of presentation and recall were the same as in Experiment 1, except that a single list of 27 TBR words (generated and tested by the method described above) was used.

The instructional manipulation was intended to differentially affect the "depth of processing" (Craik \& Lockhart, 1972) of the materials. Following the standard instructions used in Experiment 1, subjects were (1) given no further study instructions, (2) asked to generate a rhyme to the TBR word, (3) asked to generate a sentence containing the TBR word, or (4) asked to form a visual image of the TBR word. An example of the appropriate study strategy was described for all but the no-instruction group using a single nonhomographic word.

\section{Results}

Protocols were scored in a "lenient" fashion, as in Experiment 1, that is, items were counted as correct 
Table 3

Lists of TBR Homographs and Modifiers

Used in Experiment 2

\begin{tabular}{lll}
\multicolumn{1}{c}{ Meaning 1 } & \multicolumn{1}{c}{ Meaning 2 } & Homograph \\
\hline bottomless, deep & cherry, peach & PIT \\
river, mud & savings, piggy & BANK \\
human, minority & car, horse & RACE \\
boat, ship & shuffed, card & DECK \\
balance, supporting & laser, light & BEAM \\
crow, jail & drinking, singles' & BAR \\
treasure, hope & big, flat & CHEST \\
fireman's, dance & base, foot & BALL \\
social, country & caveman's, billy & CLUB \\
walking, wooden & sugar, candy & CANE \\
head, rubber & rock, jazz & BAND \\
cooling, blowing & movie, sports & FAN \\
doorbell, telephone & engagement, wedding & RING \\
U.S., money & spear, pepper & MINT \\
pig, play & fountain, ink & PEN \\
sheer, panty & fire, garden & HOSE \\
law, court & brief, suit & CASE \\
goodbye, hand & tidal, ocean & WAVE \\
traffic, log & grape, strawberry & JAM \\
Florida, tropical & sweaty, clammy & PALM \\
duck, bird & electric, gas & BILL \\
rain, water & paint, second & COAT \\
hard, knockout & hawaian, fruit & PUNCH \\
freezing, ice & nose, head & COLD \\
eye, dilated & smart, studious & PUPIL \\
clothes, steam & cast, metal & IRON \\
salad, cereal & rose, orange & BOWL \\
\hline
\end{tabular}

irrespective of their placement on the answer sheet. The data were subjected to a 6 by 4 between-groups analysis of variance, and all tests reported here are significant at or beyond the .05 level (MS $=7.86$ items, $\operatorname{LSD}_{\boldsymbol{a}=.05}=2.77$ items). The recall scores for each of the 24 groups in Experiment 2 are presented in Table 4. An overall six by four analysis of variance showed that there were main effects of instructions $[\mathrm{F}(3,168)=9.98]$ and cuing conditions $[\mathrm{F}(5,168)=$ 80.30], and that instructions and cuing conditions interacted $[F(15,168)=1.78]$. Since there are so many means in this table, and therefore so many potential comparisons, we undertook to collapse the data. It should be noted that the direction we took in collapsing the data was only one of a very large number possible. We present the group means in Table 4 so that the interested reader may proceed with his/her own data summary.

The purpose of the instructional manipulation was to differentially affect the "levels of processing," that is, increase memory through greater involvement of semantic memory, among the groups. In particular, it was hypothesized that the sentence and imagery instructions should produce "deeper" processing. This is consistent with prior formulations (Craik \& Lockhart, 1972) and seems logical since, in order to accomplish either, subjects need to interpret the TBR word according to prior language habits. By this we mean that it would be impossible to deal with the task of generating a sentence containing the word APPLE, or picture an APPLE, if the subject did not know that apples are red (or green), eaten, made into pies, grown on trees, wormy, etc. On the other hand, the no-instruction condition would seem to be "shallow" in terms of levels of processing; likewise, the rhyming condition was said to be shallow since it is generally accepted that the acoustic information generated by this type of task is not of the sort that facilitates long-term retrieval. It may be noted that one might conceptualize this manipulation as one affecting the degree of elaboration of a stimulus rather than the depth of processing.

The first direction of our analysis of Table 4, then, was toward testing the adequacy of this a priori classification of instructional condition. Within the "deep" conditions there was no effect of instructional conditions (sentence vs. imagery) $[\mathrm{F}(1,168)=2.57]$, nor was there an interaction of Instructions by Cuing Conditions $(F<1)$. For this reason, the sentence and imagery conditions were pooled for later comparisons with the no-instructions and rhyming conditions. These latter "shallow" instructions did differ from one another with respect to the level of recall $[(1,168)=11.47]$, but they did not differentially affect performance in the various cuing conditions $[F(5,168)=1.71]$. They, too, were therefore pooled, and an analysis of shallow vs. deep processing was pursued.

The deep processing instructions led to higher recall than did the shallow processing instructions $[F(1,168)=15.92]$, and processing level interacted with cuing conditions $[F(5,168)=3.47]$. Further comparisons within this interaction effect showed that shallow levels of processing, which include standard cuing instructional conditions, did not produce an advantage of same-meaning over different-meaning cues $[F(1,168)=1.15]$, while deeper levels did $[F(1,168)=21.78]$. The interaction among levels of processing and the SW, SM, and DM cuing conditions is displayed in Figure 1.

In addition to showing that the effects predicted by a semantic interpretation of encoding specificity may be obtained with certain instructions, the major cuing findings of Experiment 1 were replicated. Cuing with the same word (Condition SW) was far more effective than cuing with a different word biasing

Table 4

Mean Recall Scores (Number of Words Recalled) for Six Cuing and Four Instructional Conditions in Experiment 2

\begin{tabular}{lrrrrrr}
\hline & \multicolumn{6}{c}{ Cuing Conditions } \\
\cline { 2 - 7 } & SW & \multicolumn{1}{c}{ SM } & DM & C-0 & \multicolumn{1}{c}{$0-\mathrm{C}$} & \multicolumn{1}{c}{$0-0$} \\
\hline No Instruction & 20.38 & 9.63 & 8.50 & 9.50 & 9.88 & 11.50 \\
Rhyming & 18.00 & 9.00 & 8.00 & 9.50 & 5.63 & 7.63 \\
Sentence & 21.00 & 11.00 & $\mathbf{6 . 8 8}$ & 8.50 & 10.88 & 12.25 \\
Imagery & 21.25 & 12.75 & 7.63 & 9.88 & 11.63 & 12.88 \\
\hline
\end{tabular}




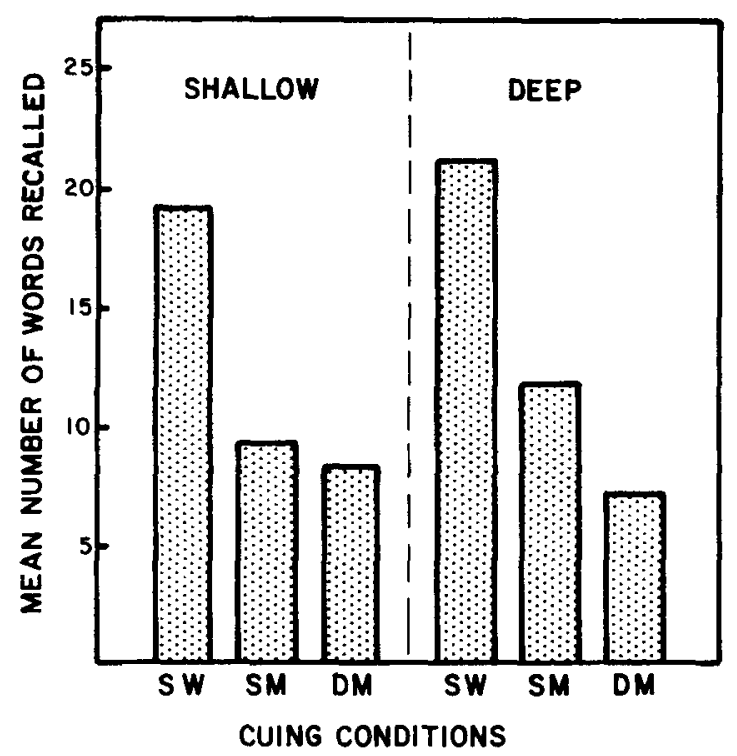

Figure 1. Recall as a function of cuing condition for "deep" and "shallow" level-of-processing instructions.

the same meaning (Condition SM) $[F(1,168)=186.19]$, but this effect did not interact with levels of processing $(\mathrm{F}<1)$. This indicates that the advantage of deep processing over shallow $[F(1,168)=10.31]$ was constant across SW and SM cuing. Also, as in Experiment 1, Condition 0-0 was superior to the pooled performance of the changed-context conditions (SM, $\mathrm{DM}, \mathrm{C}-0$, and $0-\mathrm{C})[\mathrm{F}(1,168)=10.16]$. However, this effect also interacted with instructional conditions in a complex way. While both sentence and imagery instructions produced strong and significant $0-0$ advantages, the effect under no instructions was only marginal $[F(1,168)=3.68, p<.06]$, and absent under rhyming instructions $(F<1)$. This latter null result might be attributable to poor subjective organization along semantic dimensions generated by processing the acoustic features of words, particularly when those words are homographic and need to be encoded semantically (see especially the poor recall in Condition $0-\mathrm{W}$ under rhyming).

\section{GENERAL DISCUSSION}

The encoding specificity principle, as developed by Tulving and his associates, has become a major theoretical tool in the analysis of memory processes. When coupled with the assumption that semantic components of TBR materials are involved in the formation of episodic memory traces, it has been capable of explaining and predicting a wide variety of phenomena (cf. Tulving \& Thomson, 1973). The question addressed by the present experiments is whether, under prototypical experimental conditions (e.g., those of Thomson \& Tulving, 1970) subjects, in fact, use semantic information to encode material. A semantic interpretation clearly predicts that cues relating to the same meanings of words should be more effective retrieval aids than those related to different meanings. We have shown in both of our experiments, however, that, with the usual cued recall instructions to subjects, same-meaning cues are not more effective than different-meaning cues and that both types of cues appear to interfere with "normal" retrieval patterns. This implies that little semantic processing manifests itself in the utilization of the episodic trace in the usual encoding specificity paradigm, or at least that the semantic processing occurring is small relative to that under other conditioning. This conclusion is supported by the results of Hunt and Ellis (1974) for recognition procedures.

It is interesting to note that the body of strong evidence in favor of a semantic interpretation of encoding specificity, that of Barclay et al. (1974) and of Light and Carter-Sobell (1970), was found under conditions in which the TBR material was imbedded in sentences. This, together with the finding from our own sentence and imagery conditions, strongly suggests that, while subjects are quite capable of encoding semantically, they do not unless specifically induced to do so. In addition to showing that semantic components are not normally involved in encoding specificity studies, the present experiments also contribute to our understanding of qualities of study strategies. Memory theory has increasingly been viewed from a depth-of-processing perspective (e.g., Craik \& Lockhart, 1972). The data from these experiments indicate that certain types of orienting instructions (sentences and imagery) produce semantic processing, while others (none or rhyming) do not. Through designs similar to that of Experiment 2, we should begin to investigate an additional dimension of memory processing by investigating for which levels our theories of memory are valid.

\section{REFERENCES}

Anderson, J. R., \& Bower, G. Recognition and retrieval processes in free recall. Psychological Review, 1972, 79, 97.123.

BAHRICK, H. P. Measurement of memory by prompted recall. Joumal of Experimental Psychology, 1969, 79. 213-219.

BaHRICK, H. P. A two-phase model for prompted recall. Psychological Review, 1970, 77, 215-222.

Barclay, J. R., Bransford, J. D., Franks, J. J., McCarrell. N. S., \& Nitsch, K. Comprehension and semantic flexibility. Journal of Verbal Learning and Verbal Behavior, 1974, 13, 471-481.

CRaIk, F. I. M., \& Lockhart, R. S. Levels of processing: A framework for memory research. Journal of Verbal Learning and Verbal Behavior, 1972, 11, 671-684.

Craik, F. I. M., \& Tulving, E. Depth of processing and the retention of words in episodic memory. Journal of Experimental Psychology: General, 1975, 104, 268-294. 
CRAIK, F. I. M., \& Watkins. M. J. The role of rehearsal in short-term memory. Journal of Verbal Learning and Verbal Behavior, 1973, 12. 599-607.

Cramer, P. A study of homographs. In L. Postman \& G. Keppel (Eds.), Norms of word associations. New York: Academic Press, 1970.

Ehrlich, S., \& Phillippe, M. Encoding specificity, retrieval specificity, or structural specificity? Journal of Verbal Learning and Verbal Behavior, 1976, 15, 537-548.

Hunt, R. R., \& Ellis. H. C. Recognition memory and degree of semantic contextual change. Journal of Experimental Psychology, 1974, 103, 1153-1159.

Light, L. L., \& Carter-Sobell, L. Effects of changed semantic context on recognition memory. Journal of Verbal Learning and Verbal Behavior, 1970, 9, 1-11.

MARTIN, E. Generation-recognition theory and the encoding specificity principle. Psychological Review, 1975, 82, 150-153.

Reder, L. M., Anderson, J. R., \& Bjork, R. A. A semantic interpretation of encoding specificity. Journal of Experimental Psychology, 1974, 102, 648-656.

SANTA, J. L., \& LaMwers, L. L. Encoding specificity: Fact or artifact. Joumal of Verbal Leaming and Verbal Behavior, 1974, 13, 412-423.

Thomson, D. M., \& Tulving, E. Associative encoding and retrievals: Weak and strong cues. Journal of Experimental Psychology, 1970, 86, 255-262.

Túving, E. Recall and recognition of semantically encoded words. Journal of Experimental Psychology, 1974, 102, 778-787.

Tulving, E., \& Bower, G. H. The logic of memory representations. In G. H. Bower (Ed.), The psychology of learning and motivation: Advances in research and theory (Vol. 8). New York: Academic Press, 1974.

Tulving, E., \& Thomson, D. M. Encoding specificity and retrieval processes in episodic memory. Psychological Review, 1973, 80, 352-373.
Tulving, E., \& Watkins, M. J. Structure of memory traces. Psychological Review, 1975, 82, 261-275.

WhITFORD, H. C. A dictionary of American homophones and homographs. New York: Teacher's College Press, Columbia University, 1966.

Wiseman, S., \& Tulving, E. A test of confusion theory of encoding specificity. Journal of Verbal Learning and Verbal Behavior, 1975, 14, 370-381.

WoOdward, A. E., BjoRk, R. A., \& JoNGEWARD, R. H. Recall and recognition as a function of primary rehearsal. Journal of Verbal Learning and Verbal Behavior, 1973, 12. 608-617.

\section{NOTE}

1. The list of homographs we used in Experiment 2 has occasioned comment by journal reviewers on several occasions. The comments revolve around the personal assessment of the reader as to the degree to which specific pairs of adjectives actually bias the same meaning of a TBR or the degree to which cues in the lists remain homographic to each other. We would argue that there will never be agreement on these points since in a very real sense every word in the language is related to every other word. The lists presented in Table 3 are operationally defined to meet the properties stated. We maintain that, while it is perfectly appropriate to argue that these operational definitions are inadequate, it is not reasonable to argue that the lists are inadequate when that judgment is based on one person's perception of the lists.
(Received for publication July 26, 1977; accepted October 11, 1977.) 